\title{
Interactive comment on "Possible groundwater dominance in the subglacial hydrology of ice sheet interiors: example at Dome C, East Antarctica” by Brad T. Gooch et al.
}

Anonymous Referee \#2

Received and published: 9 September 2016

\section{Summary of the paper}

The manuscript presents a model study of groundwater and subglacial water flow in East Antarctica. The authors use a 2D steady state groundwater flow model which is coupled (one way) to a 1D subglacial Weertman-sheet model. They assess the relative importance of groundwater vs subglacial flow and show that for settings such as East Antarctica, groundwater flow may dominate. They discuss their findings with respect to subglacial lakes and impacts on radar echo soundings.

This is interesting and timely research. However, the manuscript suffers from a number of mathematical shortcomings. These need to be rectified and may well impact the 
conclusions. After these problems have been addressed and probably after another round of reviews, this manuscript will be well suited for publication in TC.

\section{Main, mathematical shortcomings}

Both the groundwater flow model, the subglacial flow model and their coupling suffer from shortcomings. The groundwater model equation ( $\mathrm{Eq} 1)$ is wrong, it should read (also simplified as much as possible):

$$
\nabla \cdot\left(k \nabla\left(P+\rho_{w} g z\right)\right)=0 .
$$

The errors in the manuscript are, that the gradient in their Eq 1 was only applied to the pressure $P$, and that it needs to be $z$, the vertical coordinate, and not $z_{b}$ the elevation of the bedrock surface. Also $\mu$ and the minus sign can be dropped by multiplying by $-\mu$. I suspect that authors did solve the correct equation as it is pre-programmed in the COMSOL software which they used, but this needs confirmation.

Further they need to be specific on what their left hand side boundary condition (BC) is, they only state (line 160) it is a constant head boundary but not what that constant is. In fact, Figure 6 suggests that it is a no-flow BC, as the streamlines do not cross that boundary, and not a constant head BC. This needs clarification.

I also disagree with their choice to model subglacial lakes as part of the aquifer (line 158-160). Aquifers and lakes are on the opposite end of the permeability spectrum; solving for lake circulation using Darcy's law is just wrong. Further, there is no reason to not do it right and choose the lake bottom as boundary of the aquifer as there the pressure is known, which is what is needed as BC. I strongly suggest to revise this part of the model setup.

The main reason to add the subglacial drainage model (their Eq 2), as I see it, is to get a water-mass conserving model: the pressure $\mathrm{BC}$ of the aquifer leads to water flow into 
or out of the aquifer. This water is supplied to or taken up by the subglacial water sheet ( $G$ in $\mathrm{Eq} 2$ ). The calculation of the water layer thickness $d$ should only really be seen as a by-product of the mass-conservation calculation, in particular as the constant $12 \mu$ is ill-constrained and thus the thickness also.

To emphasis the mass-conservation aspect, equation 2 should probably be written as

$$
\nabla \cdot Q=\dot{b}+G
$$

where $Q$ is the flux $\left(\mathrm{m}^{2} / \mathrm{s}\right)$ in the subglacial sheet (or as it is a 1D problem maybe the equation should be stated as $\frac{\partial Q}{\partial x}=\dot{b}+G$ ). Note that this equation is simply solved by integrating from the top: $Q=\int_{\text {top }}^{b o t t o m} \dot{b}+G \mathrm{~d} x$ with $\mathrm{BC} Q(0)=0$. As the coupling is one way only, first the aquifer can be solved independently, then using the calculated $G$ one can solve above integral (presumably not in COMSOL but Python or Matlab. This would also reduce the lengthy Appendix B, equations A1-A5.). Also note that this integration can and should be over lakes too (it only is about mass conservation, no assumption on the type of flow is made).

As a further step, one can then calculate the water layer thickness by solving

$$
q=-\frac{d^{3}}{12 \mu} \frac{\partial\left(P_{w s}+\rho_{w} g z_{b}\right)}{\partial x}
$$

for for $d$, but just in the non-lake areas. Note that above equation also corrects for the mistake in their equation 2 to not include the elevation potential at the bed of $\rho_{w} g z_{b}$. For the lakes, the difference in inflow and outflow $Q$ could be taken as an indication of whether the lake is filling or draining or stable.

This mass-conservation approach also makes it very clear that the system is ill defined whenever $d<0$, i.e. by above eq. (3), this would mean that water flows in direction of the positive head gradient, which is not possible. The $d<0$ essentially means that the aquifer is sucking water out of the sheet where there is none (if there was a net 
positive supply then $d>0$ ). In places like this, the aquifer boundary condition would need to be changed from a head to a flow $\mathrm{BC}$ with flux $=\dot{b}$. The authors fail to realise this as their Figure 5 shows: they present results with zero sheet thickness indicating that the model has broken down. Changing BCs on the fly is difficult (some iterative scheme would probably be the easiest to implement), so I suggest that the authors only use their model when pressure BC are applicable along the whole bed and state that for $k_{0}$ higher than some $k_{\text {critical }}$ sheet flow ceases in some areas and that their model breaks down. This happens when the transmisivity of the aquifer becomes big enough to conduct all the water.

All in all, a complete overhaul of the numerical model is needed to address above comments. This also entails a re-write of section 3 of the manuscript, Appendix $B$, and the Discussion (if the new results require a different qualitative interpretation).

\section{Some line-by-line comments}

50 define "active lakes"

50 define "hydraulically flat"

59 temporal or spatial change?

65-80 I think this paragraph could be left off. The hypothesis is strong enough with just the rest of the section.

74,87 I got confused by both a $k$ and $K$. Maybe rename $k$ to $\kappa$ ?

94 "without giving an ice surface expression" This is unclear, I think the authors think of a periodic filling and drainage cycle. But also constant lakes can have a surface expression: namely being flatter than elsewhere. Reformulate.

Printer-friendly version

Section 3 State somewhere that the coupling is one-way only. Also, I would write everything in terms of hydraulic potential (or head).

Discussion paper

104 "cross-sectional model domain" is unclear 
109 "Dome C" repetition

125 "hydraulic potential"

178 This is the hydraulic potential not the pressure

387 Belongs to Discussion

435 new paragraph

435 I think the filling should be done with lakes: where the hyd-pot. gradient is negative add a lake until it becomes positive. Also the smoothing is quite strong, is that much needed? Maybe less if above suggestion on how to solve the sheet is used.

Figures: there should be no titles. The descriptions are often confusing with what is meant for which panel. The lake locations should be plotted in all figures, also in the supplement.

Fig S3 crazy numbers in the colorbars

S4 I wouldn't call this volume flux but a source

Interactive comment on The Cryosphere Discuss., doi:10.5194/tc-2016-141, 2016. 